\title{
Atomistic mechanisms governing structural stability change of zinc antimony thermoelectrics
}

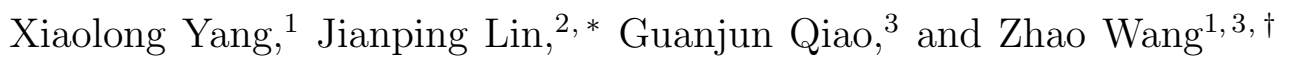 \\ ${ }^{1}$ Frontier Institute of Science and Technology, \\ Xi'an Jiaotong University, 710054, Xi'an, China. \\ ${ }^{2}$ School of Materials Science and Engineering, \\ Xiamen University of Technology, Xiamen, 361024, China. \\ ${ }^{3}$ State Key Laboratory for Mechanical Behavior of Materials, \\ Xi'an Jiaotong University, 710049, Xi'an, China
}

\begin{abstract}
The structural stability of thermoelectric materials is a subject of growing importance for their energy harvesting applications. Here we study the microscopic mechanisms governing the structural stability change of zinc antimony at its working temperature, using molecular dynamics combined with experimental measurements of the electrical and thermal conductivity. Our results show that the temperature-dependence of the thermal and electrical transport coefficients is strongly correlated with a structural transition. This is found to be associated with a relaxation process, in which a group of Zn atoms migrated between interstitial sites. This atom migration gradually leads to a stabilizing structural transition of the crystal framework, then results in a more stable crystal structure of $\beta-\mathrm{Zn}_{4} \mathrm{Sb}_{3}$ at high temperature.
\end{abstract}

*Electronic address: jaredlin@163.com (for questions on the experimental part)

$\dagger$ †lectronic address: zwangzhao@gmail.com 
Thermoelectric materials converting heat into electric power and vice versa, are of widespread interest for applications in energy harvesting and interconnection technologies. [1] For decades most of previous studies in this field have been focusing on the strategies to improve the thermoelectric energy conversion efficiency gauged by a dimensionless figure of merit ZT. 22 [3] [4] Besides the ZT, the thermal stability is also a key requirement for applications of thermoelectric materials, [5] [6] since their performance strongly depends on the material microstructure. [3] [7] [8] Despite its importance, only have a few attempts been made to address the thermal stability issue of thermoelectrics at their elevated working temperature. [9] [10] [11] [12]

Zinc antimony is a typical 'phonon glass, electron crystal', one of the best thermoelectrics at moderate temperature. [13] [14] [15]In our previous experiments, it was found that $\beta-\mathrm{Zn}_{4} \mathrm{Sb}_{3}$ samples become metastable around $425 \mathrm{~K}$, but above $565 \mathrm{~K}$ it recovers its stability. [16] Our previous simulation results showed that the diffusion of Zn atoms above $425 \mathrm{~K}$ brings remarkable anharmonicity to the system vibration, and therefore is responsible for the low thermal conductivity of $\mathrm{Zn}_{4} \mathrm{Sb}_{3}$ in the order of those found in amorphous solids. [17] However, the physical reasons behind the structural stability change remained as a open question, despite this is another key to its excellent thermoelectric performance at moderate temperature.

Thus in the present work, we try to look into this issue from an atomistic point of view using molecular dynamics (MD) simulations combining with experimental measurements on the electrical and thermal conductivities of $\beta-\mathrm{Zn}_{4} \mathrm{Sb}_{3}$. We started by building a complex hexagonal structure that corresponds to the conventional pristine structure of $\beta-\mathrm{Zn}_{4} \mathrm{Sb}_{3}$ with a concentration of $\mathrm{Zn}$ equal to 54.55 at\%. However, this structure has been found to be thermally unstable in experiments. [18] In order to obtain more realistic structure of the sample, [15] [14] [19] three Zn atoms were randomly inserted into interstitial positions at each unit cell. This yields a Zn concentration of 56.52 at\%, that has been found to avoid phase separation. [20] In our simulations we employed the classical parallel molecular dynamics package LAMMPS.[21] The simulation box comprised $12 \times 12 \times 12$ monoclinic unit cells with 14904 atoms (containing $3888 \mathrm{Sb}_{1}$ atoms, $2592 \mathrm{Sb}_{2}$ atoms, $7583 \mathrm{Zn}_{1}$ atoms and $841 \mathrm{Zn}_{2}$ atoms), with periodic boundary conditions applied in all three spatial directions. The equations of motion were integrated using the velocity Verlet algorithm with a time step of $0.5 \mathrm{fs}$. The simulations were performed in the isothermal-isobaric ensemble (NPT) 
with the Nosé-Hoover thermostat helping the system reach thermal equilibrium at different temperatures before any statistical analysis was performed. Atomistic interactions were described by a pairwise potential that has been successfully applied to study mechanical [22] and phononic behaviors of $\mathrm{Zn}_{4} \mathrm{Sb}_{3}$ [17]. Benchmark runs were performed to reproduce the characteristic $\beta$ to $\alpha$ phase transition around $250 \mathrm{~K}$, which manifests itself as a sharp step in the potential energy during a cooling process.

In our experiments, a group of $\beta-\mathrm{Zn}_{4} \mathrm{Sb}_{3}$ samples were prepared by plasma activated sintering system (PAS) with a starting Zn composition of 57.14 at\%. The compounds were prepared from 99.999 at\%-purity zinc shots and 99.99 at\%-purity antimony powder(Sinpharm Chemical Reagent Co.Ltd). These elements were loaded into a quartz ampoule and sealed under vacuum down to $10^{-4}$ torr. After sealing, the ampoules were placed in a box furnace and heated to $1023 \mathrm{~K}$ at a rate of $375 \mathrm{~K} /$ hour and kept at that temperature for 72 hours. Finally, they were quenched in cold water. The obtained ingots were ground in an agate mortar and sieved. Powders of upto $48 \mu \mathrm{m}$ diameter were loaded into graphite dies and sintered under vacuum ( $\sim 5 \mathrm{~Pa})$ using PAS at a pressure of $100 \mathrm{MPa}$ and $723 \mathrm{~K}$ for 5 minutes. The heating rate of PAS is $400 \mathrm{~K} / \mathrm{min}$. The density $(\rho)$ of the sintered samples were measured using the conventional Archimedes principle and were found to be $99 \%$ of its theoretical value $\left(6.36 \mathrm{~g} / \mathrm{cm}^{3}\right)$. The sintered samples with a $20 \mathrm{~mm}$ diameter were cut into several pieces for physical measurements using a spark erosion cutter. Rectangular bars with 12x3x3 $\mathrm{mm}$ and disks with $12.7 \mathrm{~mm}$ diameter were used for electrical transport measurements and thermal diffusivity measurements, respectively. Electrical conductivities $(\sigma)$ and Seebeck coefficients were measured simultaneously by dc four-terminal method using a Linseis Seebeck Coefficient/Electric Resistance Measuring System (LSR-3) under a lowpressure helium atmosphere from room temperature to $760 \mathrm{~K}$. Thermal diffusivities $(\alpha)$ and specific heats $\left(C_{p}\right)$ were obtained using a laser flash apparatus (NETZSCH LFA 457). The samples were coated with a thin layer of graphite to minimize errors from the emissivity of the material. The thermal conductivity $(\lambda)$ was calculated from the relationship: $\lambda=\rho \alpha C_{p}$, where $\rho$ is the mass density.

In our simulation results, we obtained the crystal lattice volume and the potential energy at increasing temperature. In Fig, 1, we can see a volume decrease when the temperature reaches about $425 \mathrm{~K}$, accompanied with changes in the temperature dependence of the experimentally measured electrical and thermal conductivities. In our previous studies, we 


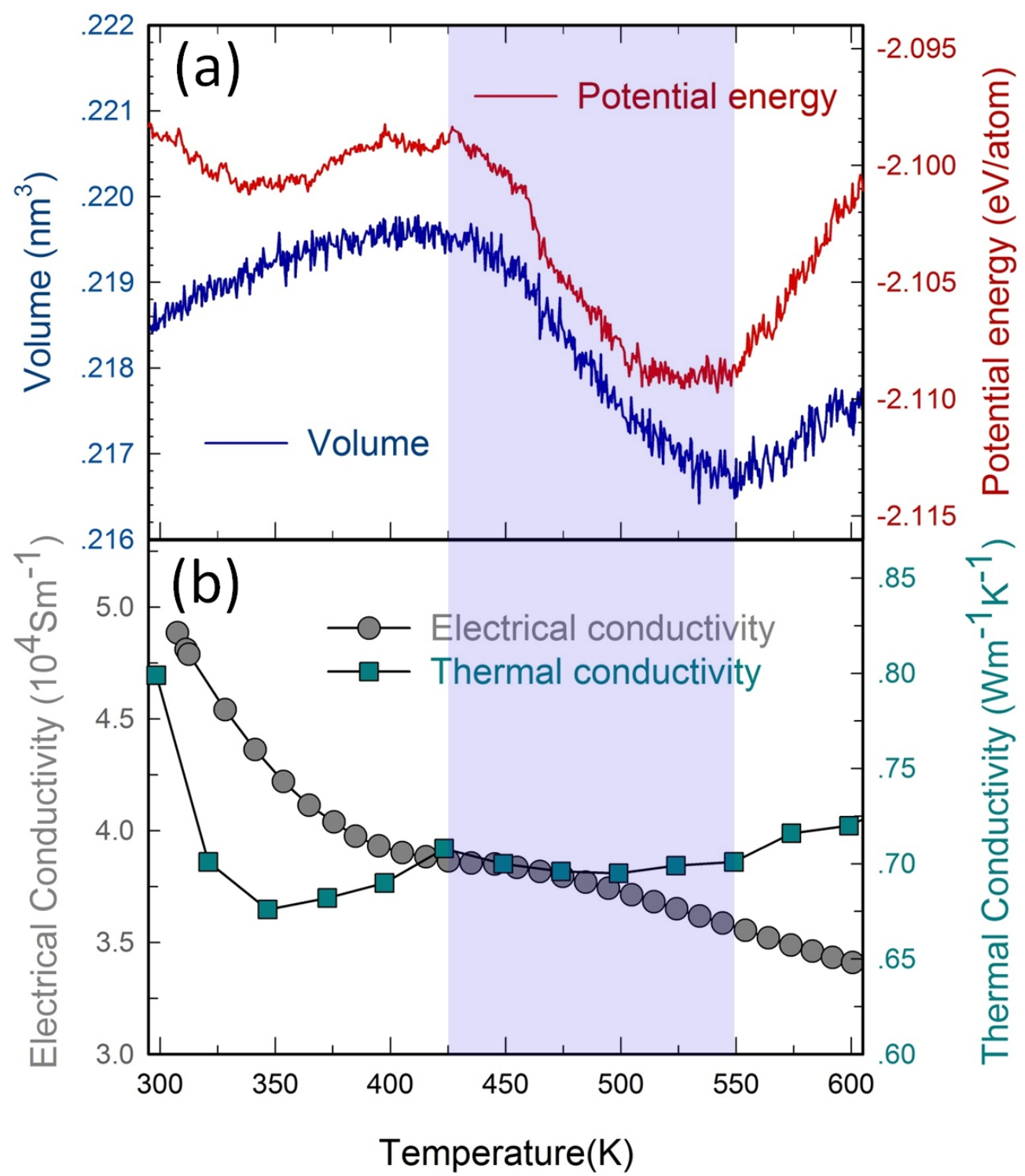

FIG. 1: (a) Volume (left ordinate axis) and potential energy (right ordinate axis) variations of $\beta-\mathrm{Zn}_{4} \mathrm{Sb}_{3}$ at increasing temperature. (b) Experimentally measured electrical and thermal conductivities of $\beta-\mathrm{Zn}_{4} \mathrm{Sb}_{3}$ at increasing temperature. The highlighted background corresponds to the temperature interval at which the structure transition takes place.

found that this volume change is triggered by a diffusion-like behavior of interstitial $\mathrm{Zn}$ atoms, this produces remarkable phonon anharmonicity responsible for the $\mathrm{Zn}_{4} \mathrm{Sb}_{3}$ low thermal conductivity.[17] Here we instead focus our attention on a different aspect, including a structural transition after this avalanche of atomic migration. The energy curve in Fig.1(a) reveals the nature of this process, in which a group of loosely bonded atoms adapts to a 
different crystallographic structure in their search for a more energetically favorable arrangement. In particular, we can see that a structural transition takes place at about $550 \mathrm{~K}$ where the relaxation process seems to be accomplished, the volume and the energy again start to increase with increasing temperature.
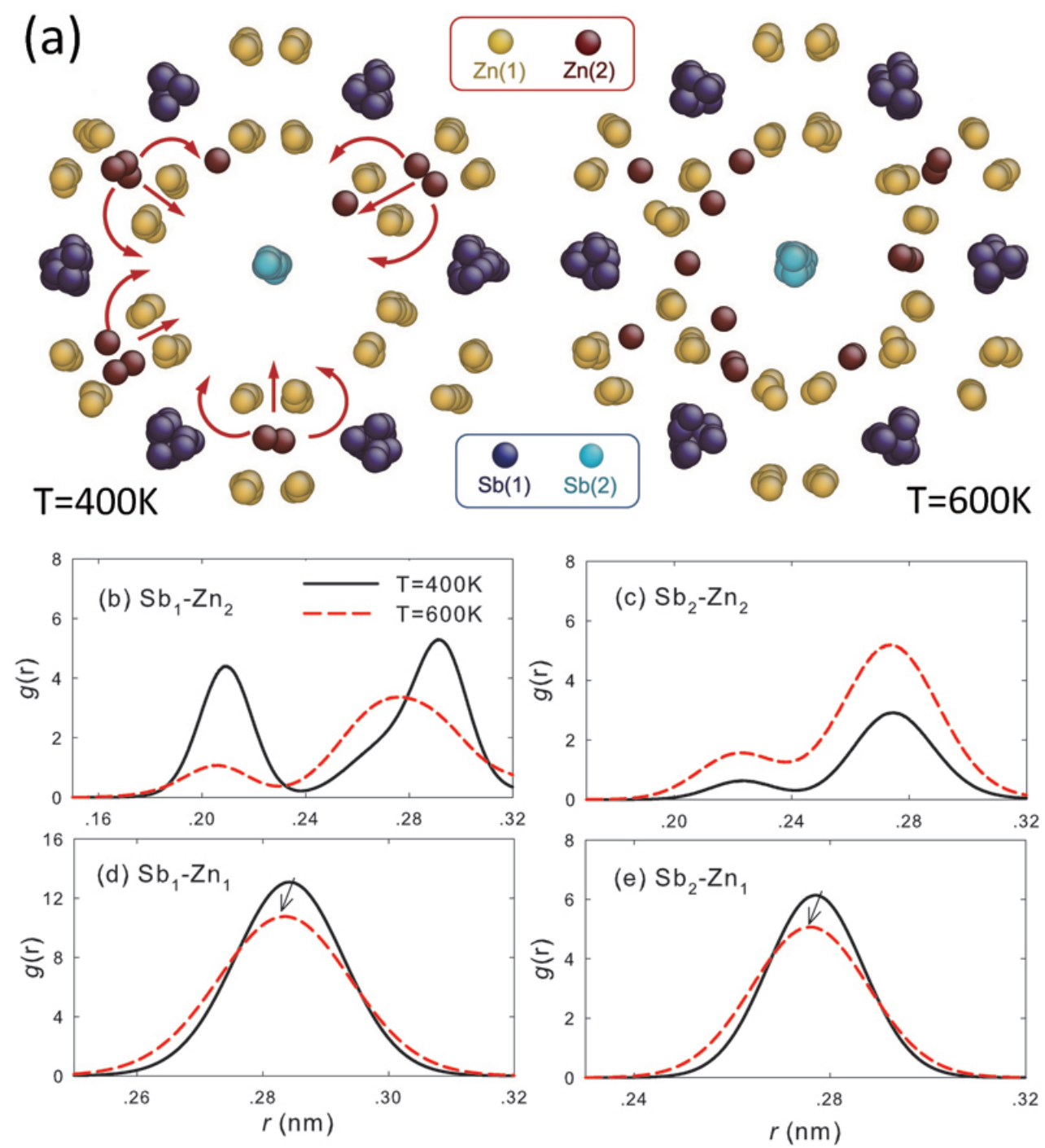

FIG. 2: (a) Atomistic configurations of $\beta-\mathrm{Zn}_{4} \mathrm{Sb}_{3}$ from $\langle 001\rangle$ before (left at $400 \mathrm{~K}$ ) and after (right at $600 \mathrm{~K}$ ) a structure transition. The positions of atoms are averaged over a period of 0.5 ns. Circles in four different colors show $\mathrm{Zn}$ atoms at crystal sites $\left(\mathrm{Zn}_{1}\right), \mathrm{Zn}$ atoms at glass-like sites $\left(\mathrm{Zn}_{2}\right)$, and $\mathrm{Sb}$ atoms at two different crystal sites $\left(\mathrm{Sb}_{1}\right.$ and $\left.\mathrm{Sb}_{2}\right)$, respectively. The arrows show possible displacement trajectories of $\mathrm{Zn}_{2}$ atoms. (b-e) Radial distribution functions $g(r)$ of different pair correlations. 
In order to obtain more information about this transition occurring around $550 \mathrm{~K}$, we have performed detailed analyses on the simulated atomistic trajectories. Fig.2(a) shows two atomistic configurations of the simulated $\beta-\mathrm{Zn}_{4} \mathrm{Sb}_{3}$ before (left panel) and after (right panel) the structure transition. Interstitial $\mathrm{Zn}$ atoms $\left(\mathrm{Zn}_{2}\right)$ are found to migrate to inner sites, while $\mathrm{Zn}$ atoms at crystalline sites $\left(\mathrm{Zn}_{1}\right)$ seem to stay in their positions. Furthermore, we have calculated the radial distribution functions $g(r)$ of different pair correlations, through which the local relaxed structure associated with each atom is monitored. By comparing the case at $400 \mathrm{~K}$ with that at $600 \mathrm{~K}$, we can see that $g(r)$ of $\mathrm{Zn}_{2}-\mathrm{Sb}_{1}$ pairs becomes lower and broader at increasing temperature [Fig,2(b)], while that of $\mathrm{Zn}_{2}-\mathrm{Sb}_{2}$ pairs exhibits an inverse trend [Fig[2(c)]. It is therefore double-confirmed that $\mathrm{Zn}_{2}$ atoms migrate from outer to inner sites and become closer to $\mathrm{Sb}_{2}$ atoms. Regarding the main crystal lattice framework composed of $\mathrm{Sb}_{1}, \mathrm{Sb}_{2}$ and $\mathrm{Zn}_{1}$ atoms, which are $26.087 \%, 17.39 \%$ and $50.879 \%$ occupied in our system, respectively, we can see that $g(r)$ of $\mathrm{Sb}_{1}-\mathrm{Zn}_{1}$ and $\mathrm{Sb}_{2}-\mathrm{Zn}_{1}$ pairs [Fig.2(d,e)] are both broadened by increasing temperature after the structural transition, with peaks shifted to the left side. This shift indicates a change of interatomic distance in the main lattice, corresponding to the volume decrease during his structural transition.

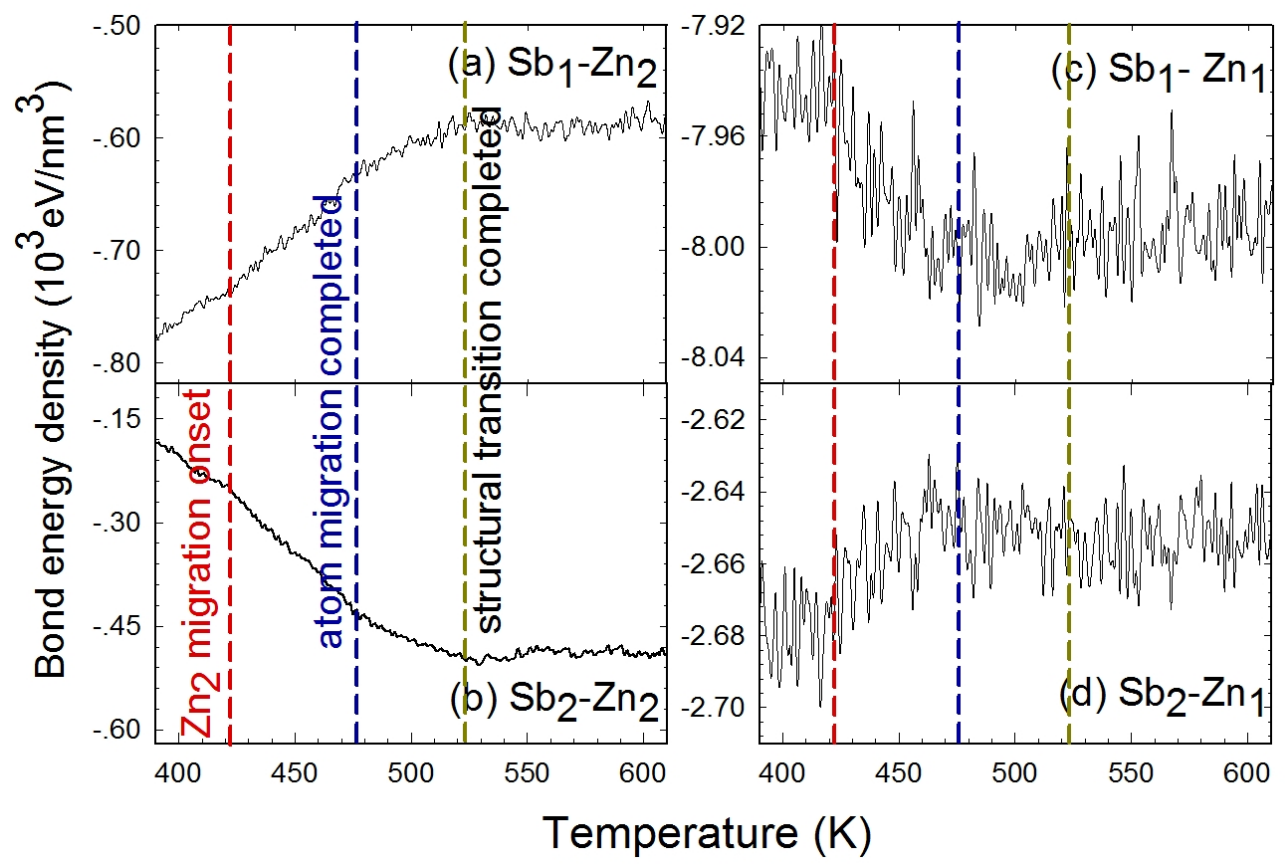

FIG. 3: Bond potential energy per unit cell volume between different types of atoms [(a)Sb 1 and $\mathrm{Zn}_{2}$, (b)Sb $\mathrm{Sb}_{2}$ and $\mathrm{Zn}_{2}$, (c)Sb and $\mathrm{Zn}_{1}$, (d) $\mathrm{Sb}_{2}$ and $\mathrm{Zn}_{1}$ ] versus the temperature. 
To confirm the above-described atomistic-scale details, we plot the pair potential energy between different atom sets in Fig.3. It can be seen that the bonds between $\mathrm{Zn}_{2}$ and $\mathrm{Sb}_{1}$ atoms is weakened at increasing temperature, while those between $\mathrm{Zn}_{2}$ and $\mathrm{Sb}_{2}$ atoms get greatly enhanced. These are consistent with the observations commented with Fig.2 The bond energy value of $\mathrm{Sb}_{1}-\mathrm{Zn}_{1}$ pair decreases while $\mathrm{Sb}_{2}-\mathrm{Zn}_{1}$ pairs increases at increasing temperature, these are also in good agreement with the above-discussed radial distribution function data.

In conclusion, we have investigated the atomistic origin of the structural stability change of $\beta-\mathrm{Zn}_{4} \mathrm{Sb}_{3}$, which is strongly correlated with its electrical and thermal transport coefficients. Our simulation results showed that the evolution of the crystallographic stability of $\beta-\mathrm{Zn}_{4} \mathrm{Sb}_{3}$ proceeds through four steps. Firstly, thermal energy fuels a long-range relaxation process, in which a fraction of $\mathrm{Zn}_{2}$ atoms start to migrate between interstitial sites at about $420 \mathrm{~K}[\mathrm{Fig} \cdot 3(\mathrm{a}, \mathrm{b})]$. Secondly, the thermally-activated atom migration gradually leads to a stabilizing structural transition of the main crystal framework composed of $\mathrm{Zn}_{1}$ and $\mathrm{Sb}$ [Fig.3(c,d)] which are $50.879 \%$ and $43.477 \%$ occupied, respectively, this process seems to be accomplished at about 480K. Thirdly, the migrated Zn atoms exhibit also a local relaxation process in which they search for a more energetically favorable configuration [Fig, $3(a, b)]$. Finally, the above-discussed processes result in a more stable crystal structure of $\beta-\mathrm{Zn}_{4} \mathrm{Sb}_{3}$ at high temperature, the system recovers it structural stability at about 520K[Fig 3 (a-d)]. This microscopic mechanism explains the physical reason why the thermoelectric performance of zinc antimony is sensible to slight variation in Zn composition. This is consistent with experimental observations [23] and is applicable to super-ionic frameworks in large lattice (e.g. $\mathrm{Sb}$ frames) with relatively small-radius interstitials with high diffusion mobility.

We thank Dr. J. Carrete at CEA grenoble and Prof. J. Li at MIT for helpful discussions. This work is supported by a grant-in-aid of 985 Project from Xi'an Jiaotong University, the National Natural Science Foundation of China (Grant No. 11204228), the National Basic Research Program of China (2012CB619402 and 2014CB644003) and the Fundamental Research Funds for the Central Universities.

[1] L. E. Bell, Science 321, 1457 (2008).

[2] C. J. Vineis, A. Shakouri, A. Majumdar, and M. G. Kanatzidis, Adv. Mater. 22, 3970 (2010). 
[3] Y. Pei, H. Wang, and G. J. Snyder, Adv. Mater. 24, 6125 (2012).

[4] M. Zebarjadi, K. Esfarjani, M. S. Dresselhaus, Z. F. Ren, and G. Chen, Energ. Environ. Sci. 5, $5147(2012)$.

[5] J.-F. Li, W.-S. Liu, L.-D. Zhao, and M. Zhou, NPG Asia. Mater. 2, 152 (2010).

[6] G. J. Snyder and E. S. Toberer, Nat. Mater. 7, 105 (2008).

[7] K. Biswas, J. He, I. D. Blum, Chun-Iwu, T. P. Hogan, D. N. Seidman, V. P. Dravid, and M. G. Kanatzidis, Nature 490 (2012).

[8] D. J. Singh, Phys. Rev. B 81, 195217 (2010).

[9] G. J. Snyder, M. Christensen, E. Nishibori, T. Caillat, and B. B. Iversen, Nat. Mater. 3, 458 (2004).

[10] H. Wang, A. D. LaLonde, Y. Pei, and G. J. Snyder, Adv. Funct. Mater. 23, 1586 (2013).

[11] J. Xu, J. Tang, K. Sato, Y. Tanabe, H. Miyasaka, M. Yamashita, S. Heguri, and K. Tanigaki, Phys. Rev. B 82, 085206 (2010).

[12] H. Yin, B. L. Pedersen, and B. B. Iversen, Eur. J. Inorg. Chem. 2011, 2733 (2011).

[13] T. Caillat, J. P. Fleurial, and A. Borshchevsky, J. Phys. Chem. Solids 58, 1119 (1997).

[14] J. Nylen, M. Andersson, S. Lidin, and U. Haussermann, J. Am. Chem. Soc. 126, 16306 (2004).

[15] E. S. Toberer, P. Rauwel, S. Gariel, J. Tafto, and G. J. Snyder, J. Mater. Chem. 20, 9877 (2010).

[16] J. Lin, X. Li, G. Qiao, Z. Wang, J. Carrete, Y. Ren, L. Ma, Y. Fei, B. Yang, L. Lei, and J. Li, J. Am. Chem. Soc. 136, 1497 (2014).

[17] X. Li, J. Carrete, J. Lin, G. Qiao, and Z. Wang, Appl. Phys. Lett. 103, 103902 (2013).

[18] V. Izard, M. C. Record, and J. C. Tedenac, J. Alloys Compd. 345, PII S0925 (2002).

[19] E. S. Toberer, K. A. Sasaki, C. R. I. Chisholm, S. M. Haile, I. Goddard, W. A., and G. J. Snyder, Phys. Status Solidi. 1, 253 (2007).

[20] P. Rauwel, O. M. Lovvik, E. Rauwel, E. S. Toberer, G. J. Snyder, and J. Tafto, Phys. Status Solidi. 208, 1652 (2011).

[21] S. Plimpton, J. Comput. Phys. 117, 1 (1995).

[22] G. Li, Y. Li, X. Yang, Y. Tong, A. Zhou, L. Liu, and P. Zhai, J. Electron. Mater. 40, 1158 (2011).

[23] B. L. Pedersen, H. Yin, H. Birkedal, M. Nygren, B. B. Iversen, Chem. Mater. 22, 2375 (2010). 